\title{
Proinflammatory cytokines in Parkinson's disease and current treatment approaches
}

\author{
Berk Ahmet * \\ Elazı̆̆ Fethi Sekin City Hospital 23050-Elazı̆̆, Turkey.
}

Publication history: Received on 15 October 2019; revised on 17 December 2019; accepted on 23 December 2019

Article DOI: https://doi.org/10.30574/gscarr.2020.2.1.0012

\begin{abstract}
Parkinson's disease is a neurodegenerative disease characterized by progressive degeneration of dopaminergic neurons in the substantia nigra region. As the etiology is not clear, treatment in this disease is rather symptomatic and inadequate to prevent the progression of the disease. However, recent studies have demonstrated the strong association of neuroinflammation and proinflammatory cytokines with disease pathology. In this review, the relationship of proinflammatory cytokines with the pathology of Parkinson's disease is discussed and current treatment approaches targeting these mechanisms are evaluated.
\end{abstract}

Keywords: Current approaches; Neurodegenerative disease; Neuroinflammation; Parkinson's disease; Proinflammatory cytokines

\section{Introduction}

Parkinson's disease is a progressive nervous system disorder, typically a disease of middle and older ages, starting at an average age of 50-60 years and progressively progressing over a period of about 10-20 years. The prevalence of Parkinson's was reported to be over $1 / 1000$ and $2 \%-3 \%$ of patients over 65 years of age were found to have the disease [1]. A number of studies have shown that neuroinflammation plays an important role in the pathogenesis of Parkinson's disease [2]. In particular, proinflammatory cytokines, including interleukin-1 beta (IL1 $\beta$ ), interleukin-6 (IL-6), interleukin-2 (IL-2), and tumor necrosis factor alpha (TNF- $\alpha$ ) have been proposed to be part of an immune response to tissue damage. Clinical, preclinical and therapeutic studies support the presence of a neuroinflammatory cascade in Parkinson's disease as in other neurodegenerative diseases [3, 4]. Activated microglia cells were detected with increased expression of proinflammatory mediators in the substantia nigra regions of patients with Parkinson's disease [5]. Studies have shown that blood IL-1ß levels are significantly higher in patients with Parkinson's disease [6]. Inflammation may contribute to the development of both motor and non-motor symptoms [7]. Abnormal processing by ubiquitin - proteasome and autophagy- lysosomal systems of misfolded proteins, oxidative stress, mitochondrial dysfunction, inflammation and other pathogenic mechanisms have been reported to cause the death of dopaminergic and non-dopaminergic cells in the brains of Parkinson's patients [8].

The main problem in Parkinson's disease is the development of motor symptoms after the loss of more than half of nigrostrial dopaminergic neurons. This makes the treatment difficult because the disease is not noticed early and causes the treatment to be started on time. Another problem is that treatment approaches are inadequate because the pathology of the disease is not clarified. Drug therapy in Parkinson's disease is symptomatic and aims to relieve symptoms. In the later stages of the disease, in which neuronal loss is further increased, the addition of new drugs to the treatment or increasing the dose of the available drugs significantly increases the drug-related side ethers. Inadequate treatment, high cost and serious side effects in Parkinson's disease require new treatment approaches. A

\footnotetext{
${ }^{*}$ Corresponding author

E-mail address: eczaberk@gmail.com
} 
thorough understanding of the etiology of the disease is important for new therapies to be successful. In this review, the relationship between proinflammatory cytokines and Parkinson's disease is examined and current treatment approaches are discussed. The role of neuroinflammation in disease pathology is emphasized.

\section{New pathways and targets associated with cytokines in Parkinson's disease}

Microglial activation is one of the main causes of neuroinflammation in Parkinson's disease, and microRNAs (miRs) are key factors in this mechanism. One of them, overexpression of miR-195, suppresses pro-inflammatory cytokines secretion such as IL-6 and TNF- $\alpha$ and increases anti-inflammatory cytokines secretion such as IL-4 and IL-10. Rhoassociated kinase 1 (ROCK1) pathway plays an important role in this mechanism and constitutes an important target for Parkinson's disease [9]. It has been suggested that miR-21 downregulation inhibiting 1-Methyl-4phenylpyridinium-mediated toxicity by reducing pro-inflammatory cytokines [IL -6 , IL-1 $\beta$ and TNF- $\alpha$ ] and reactive oxygen species (ROS) production, may be a new target in Parkinson's disease [10].

In another study, downregulation of the protein kinase-1 and -3 / mixed lineage kinase domain-like protein (RIP1 / RIP3 / MLKL) pathway was shown to improve 1-methyl-4-phenyl-1,2,3,6-tetrahydro pyridine (MPTP) induced Parkinson's disease via inflammatory cytokines and dopaminergic neurons, and these genes were reported to be effective in disease pathology [11].

The immune response seen in Parkinson's disease is not only due to microglial activation in the brain, but also changes in the peripheral immune system. In one study, it was observed that blood monocytic of patients with Parkinson's disease may be unresponsive to specific stimulations and showed a shorter viability [12].

The receptor for advanced glycation endproducts RAGE, involved in the modulation of inflammation, has been found to be effective in neuroinflammation and dopaminergic neuron loss in Parkinson's disease and is thought to be a target for treatment [13]. It is thought that G protein-coupled estrogen receptor (GPER), which is known to mediate neuroptoactive effect, can be used against the progression of parkinsonism with its anti-neuroinflammatory effect [14]. Cerebral hypoperfusion is also thought to increase cognitive impairment by increasing neuroinflammation in Parkinson's disease [15].

Recently, studies targeting cannabinoid receptors have also been conducted. $\beta$-caryophyllene (BCP), a naturally occurring cannabinoid type two receptors (CB2) receptor agonist, is a potential therapeutic agent that exhibits antioxidant and anti-inflammatory effects and is mediated by cannabinoid type two receptors (CB2) activation [16].

A newly-identified proinflammatory cytokine, interleukin (IL)-32 was found to worsening MPTP neurotoxicity by inducing neuroinflammation [17]. Another cytokines IL-4 and IL-13 have been suggested to have an effect on cognitive functions by stimulation of astrocytes and attention was drawn to the relationship with dementia due to Parkinson's disease [18]. In a study examining the relationship of air pollution and genetic factors with parkinson's disease, it was noted that traffic-related air pollution and genetic variation in the proinflammatory cytokine gene IL1B may pose a risk for disease development [19].

Parkinson's disease is also closely associated with depression, and approximately 20\% of patients with Parkinson's develop depressive disorders [20]. Considering that proinflammatory cytokines play a role in the pathophysiology of both depression and Parkinson's disease, we think that the approaches targeting this pathway will play an important role in both the treatment of the disease and the elucidation of the etiology of the disease.

\section{Current treatment approaches associated with cytokines}

Aggregated forms of the synaptic protein $\alpha$-synuclein $(\alpha S)$ plays a role in Parkinson's disease by triggering microglial inflammatory process and neurodegradation. In a study of microglial cell culture induced by recombinant human $\alpha \mathrm{S}$, the pretreatment effect of rifampicin and rifampicin quinone was evaluated. Rifampicin and rifampicin quinone inhibited PI3K- and non-PI3K signaling pathways and reduced microglial activation. The neuroprotective effect of rifampicin quinone was found to be more potent than rifampicin, and PI3K inhibition was thought to be particularly important in the control of oxidative stress and it was suggested that Rifampicin quinone was promising as an antiparkinsionian drug [21].

Hypoxia-inducible factor-1 alpha (HIF-1 $\alpha$ ) and nuclear receptor related-1 (Nurr1) genes play a critical role in the survival of dopaminergic neurons, and disorders in these genes may play a role in Parkinson's pathology. Albendazole, 
an anthelminthic benzimidazoles known to provide Nurr1 activation, showed neuroprotective effect by increasing the expression of these genes in the rotenone-induced Parkinson model. The neuroprotective effect of albendazole is thought to be effective in improving strial dopamine levels and motor functions in the substantia nigra and suppressing the expression of neurotoxic proinflammatory cytokines [22].

In-vitro farrerol reduced the production of lipopolysaccharide (LPS) -induced inflammatory mediators such as IL-6, IL$1 \beta$, TNF- $\alpha$, cyclooxygenase 2 (COX-2) in BV-2 cells and increased nitric oxide synthase (iNOS). This mechanism is thought to be caused by inhibiting NF- $\kappa$ B p65 and AKT phosphorylation. Furthermore, farrerol demonstrated neuroprotective effect by reducing microglial activation and dopaminergic cell death in the LPS-induced rat PD model and it was thought to be promising for Parkinson's disease [23].

A selective estrogen receptor modulator, raloxifene, has been shown to be a promising drug for the treatment of early Parkinson's disease by preventing systemic inflammation and peripheral neuronal dysfunction [24].

Conventional drugs provide symptomatic relief in Parkinson's disease and inadequate progression of the disease has led to the idea of developing treatment with natural substances. For this purpose, Phloretin was tested on MPTP induced PD in mice model. MPTP-induced reduced dopamine (DA) levels, motor abnormalities, reduced tyrosine hydroxylase $(\mathrm{TH})$ enzyme protein expression and inflammation were significantly improved by phloretin. Improvements in levels of specific inflammatory markers like glial fibrillary acidic protein (GFAP), proinflammatory cytokines such as IL- $\beta$, IL-6, and TNF- $\alpha$ have been proposed to play an important role in the neuroprotective effect of phloretin and treatment has been reported to be promising for Parkinson's disease [25].

Another study on the MPTP induced Parkinson's disease in mice model showed that thymoquinone increased antioxidant enzyme levels, prevented lipid peroxidation, and improved pro-inflammatory cytokines. Thymoquinone also provided an increase in the level of inflammatory parameters such as COX-2 and iNOS, furthermore it prevented cell death stimulated by $\alpha$-synuclein aggregation and pre-formed fibrils [26]. Thymol, a dietary monoterpene phenol, showed a neuroprotective effect in the rotenone (ROT) -induced Parkinson model by maintaining the endogenous antioxidant defense mechanism and reducing inflammatory mediators including cytokines and enzymes [27].

Nerolidol, a sesquiterpene alcohol, showed neuroprotective effect in rotenone-induced experimental model of PD due to its antioxidant and anti-inflammatory properties [28].

Acetyl-L-carnitine has been shown to have a therapeutic effect against neurodegenerative diseases in rat models, which is thought to play a role in balancing the proinflammatory system [29].

Baicalein, a major bioactive flavone, showed antiparkinsonian effect in animal models by suppressing the production of proinflammatory cytokines, regulating arthrocytes and microglial activation, and suppressing the nuclear factor- $\mathrm{K} B$ (NF-кB) pathway [30].

It is thought that curcumin, which has antioxidant and anti-inflammatory properties, can be a drug candidate in Parkinson's disease by preventing $\alpha$-synuclein aggregates in the dopaminergic neurons [31].

In a study examining the effect of probiotics on the patients with Parkinson's disease, it was found that especially Lactobacillus salivarius LS01 and L. acidophilus decreased the level of pro-inflammatory cytokines and increased the level of anti-inflammatory cytokines, and treatment with probiotics was thought to be promising for the disease. It is noteworthy that the strains found to be successful in this study do not carry the tdc gene, and this gene is known to reduce levodopa bioavailability in patients with Parkinson's disease [32].

Interestingly, it was reported that the pathway of proinflammatory cytokines plays a role in the effect of acupuncture and electroacupuncture, an alternative treatment for Parkinson's disease [33].

\section{Conclusion}

The importance of neuroinflammation in the mechanisms of dopaminergic neuron loss, which is the main cause of Parinson's disease, has been shown in the studies. From this point of view, it is clear that focusing on, proinflammatory cytokines and neuroinflammation pathways for the treatment of Parkinson's disease will play an important role in the development of new therapies. Further studies are needed to elucidate these mechanisms. 


\section{Compliance with ethical standards}

\section{Acknowledgments}

I would like to express my gratitude to Elazı̆̆ Fethi Sekin City Hospital for its support for academic studies.

\section{Disclosure of conflict of interest}

Author hereby state that there is no conflict of interest.

\section{References}

[1] Twelves D, Perkins KS and Counsell C. (2003). Systematic review of incidence studies of Parkinson's disease. Mov Disord, 18(1), 19-31.

[2] Rocha NP, de Miranda AS and Teixeira AL. (2015). Insights into Neuroinflammation in Parkinson's Disease: From Biomarkers to Anti-Inflammatory Based Therapies. Biomed Res Int, 628192.

[3] Gao HM, Liu B, Zhang W and Hong JS. (2003). Novel anti-inflammatory therapy for Parkinson's disease. Trends Pharmacol Sci, 24(8), 395-401.

[4] Mosley RL, Benner EJ, Kadiu I, Thomas M, Boska MD, Hasan K, et al. (2006). Neuroinflammation, Oxidative Stress and the Pathogenesis of Parkinson's Disease. Clin Neurosci Res, 6(5), 261-281.

[5] Knott C, Wilkin GP and Stern G. (1999). Astrocytes and microglia in the substantia nigra and caudate-putamen in Parkinson's disease. Parkinsonism Relat Disord, 5(3), 115-122.

[6] Alrafiah A, Al-Ofi E, Obaid MT and Alsomali N. (2019). Assessment of the Levels of Level of Biomarkers of Bone Matrix Glycoproteins and Inflammatory Cytokines from Saudi Parkinson Patients. Biomed Res Int, 2690205.

[7] Barnum CJ and Tansey MG. (2012). Neuroinflammation and non-motor symptoms: the dark passenger of Parkinson's disease? Curr Neurol Neurosci Rep, 12(4), 350-358.

[8] Jankovic J. (2008). Parkinson's disease: clinical features and diagnosis. J Neurol Neurosurg Psychiatry, 79(4), 368376.

[9] Ren Y, Li H, Xie W, Wei N and Liu M. (2019). MicroRNA195 triggers neuroinflammation in Parkinson's disease in a Rhoassociated kinase 1dependent manner. Mol Med Rep, 19(6), 5153-5161.

[10] Mao H and Ding L. (2019). Downregulation of miR-21 suppresses 1-methyl-4-phenylpyridinium-induced neuronal damage in MES23.5 cells. Exp Ther Med, 18(4), 2467-2474.

[11] Lin QS, Chen P, Wang WX, Lin CC, Zhou Y, Yu LH, et al. (2019). RIP1/RIP3/MLKL mediates dopaminergic neuron necroptosis in a mouse model of Parkinson disease. Lab Invest.

[12] Nissen SK, Shrivastava K, Schulte C, Otzen DE, Goldeck D, Berg D, et al. (2019). Alterations in Blood Monocyte Functions in Parkinson's Disease. Mov Disord.

[13] Gasparotto J, Ribeiro CT, Bortolin RC, Somensi N, Rabelo TK, Kunzler A, et al. (2017). Targeted inhibition of RAGE in substantia nigra of rats blocks 6-OHDA-induced dopaminergic denervation. Sci Rep, 7(1), 8795.

[14] Guan J, Yang B, Fan Y and Zhang J. (2017). GPER Agonist G1 Attenuates Neuroinflammation and Dopaminergic Neurodegeneration in Parkinson Disease. Neuroimmunomodulation, 24(1), 60-66.

[15] Gao Y, Tang H, Nie K, Zhu R, Gao L, Feng S, et al. (2019). Hippocampal damage and white matter lesions contribute to cognitive impairment in MPTP-lesioned mice with chronic cerebral hypoperfusion. Behav Brain Res, 368, 111885.

[16] Javed H, Azimullah S, Haque ME and Ojha SK. (2016). Cannabinoid Type 2 (CB2) Receptors Activation Protects against Oxidative Stress and Neuroinflammation Associated Dopaminergic Neurodegeneration in Rotenone Model of Parkinson's Disease. Front Neurosci, 10, 321.

[17] Jung YY, Katila N, Neupane S, Shadfar S, Ojha U, Bhurtel S, et al. (2017). Enhanced dopaminergic neurotoxicity mediated by MPTP in IL-32beta transgenic mice. Neurochem Int, 102, 79-88. 
[18] Brombacher TM, Nono JK, De Gouveia KS, Makena N, Darby M, Womersley J, et al. (2017). IL-13-Mediated Regulation of Learning and Memory. J Immunol, 198(7), 2681-2688.

[19] Lee PC, Raaschou-Nielsen O, Lill CM, Bertram L, Sinsheimer JS, Hansen J, et al. (2016). Gene-environment interactions linking air pollution and inflammation in Parkinson's disease. Environ Res, 151, 713-720.

[20] Ryan M, Eatmon CV and Slevin JT. (2019). Drug treatment strategies for depression in Parkinson disease. Expert Opin Pharmacother, 20(11), 1351-1363.

[21] Acuna L, Hamadat S, Corbalan NS, Gonzalez-Lizarraga F, Dos-Santos-Pereira M, Rocca J, et al. (2019). Rifampicin and Its Derivative Rifampicin Quinone Reduce Microglial Inflammatory Responses and Neurodegeneration Induced In Vitro by alpha-Synuclein Fibrillary Aggregates. Cells, 8(8).

[22] Kandil EA, Sayed RH, Ahmed LA, Abd El Fattah MA and El-Sayeh BM. (2019). Hypoxia-inducible factor 1 alpha and nuclear-related receptor 1 as targets for neuroprotection by albendazole in a rat rotenone model of Parkinson's disease. Clin Exp Pharmacol Physiol.

[23] Li Y, Zeng Y, Meng T, Gao X, Huang B, He D, et al. (2019). Farrerol protects dopaminergic neurons in a rat model of lipopolysaccharide-induced Parkinson's disease by suppressing the activation of the AKT and NF-kappaB signaling pathways. Int Immunopharmacol, 75, 105739.

[24] Poirier AA, Cote M, Bourque M, Morissette M, Di Paolo T and Soulet D. (2016). Neuroprotective and immunomodulatory effects of raloxifene in the myenteric plexus of a mouse model of Parkinson's disease. Neurobiol Aging, 48, 61-71.

[25] Zhang G, Yang G and Liu J. (2019). Phloretin attenuates behavior deficits and neuroinflammatory response in MPTP induced Parkinson's disease in mice. Life Sci, 232, 116600.

[26] Ardah MT, Merghani MM and Haque ME. (2019). Thymoquinone prevents neurodegeneration against MPTP in vivo and modulates alpha-synuclein aggregation in vitro. Neurochem Int, 128, 115-126.

[27] Javed H, Azimullah S, Meeran MFN, Ansari SA and Ojha S. (2019). Neuroprotective Effects of Thymol, a Dietary Monoterpene Against Dopaminergic Neurodegeneration in Rotenone-Induced Rat Model of Parkinson's Disease. Int J Mol Sci, 20(7).

[28] Javed H, Azimullah S, Abul Khair SB, Ojha S and Haque ME. (2016). Neuroprotective effect of nerolidol against neuroinflammation and oxidative stress induced by rotenone. BMC Neurosci, 17(1), 58.

[29] Singh S, Mishra A, Srivastava N, Shukla R and Shukla S. (2018). Acetyl-L-Carnitine via Upegulating Dopamine D1 Receptor and Attenuating Microglial Activation Prevents Neuronal Loss and Improves Memory Functions in Parkinsonian Rats. Mol Neurobiol, 55(1), 583-602.

[30] Zhang X, Yang Y, Du L, Zhang W and Du G. (2017). Baicalein exerts anti-neuroinflammatory effects to protect against rotenone-induced brain injury in rats. Int Immunopharmacol, 50, 38-47.

[31] Sharma N and Nehru B. (2018). Curcumin affords neuroprotection and inhibits alpha-synuclein aggregation in lipopolysaccharide-induced Parkinson's disease model. Inflammopharmacology, 26(2), 349-360.

[32] Magistrelli L, Amoruso A, Mogna L, Graziano T, Cantello R, Pane M, et al. (2019). Probiotics May Have Beneficial Effects in Parkinson's Disease: In vitro Evidence. Front Immunol, 10, 969.

[33] Tamtaji OR, Naderi Taheri M, Notghi F, Alipoor R, Bouzari R and Asemi Z. (2019). The effects of acupuncture and electroacupuncture on Parkinson's disease: Current status and future perspectives for molecular mechanisms. J Cell Biochem, 120(8), 12156-12166.

\section{How to cite this article}

Berk A. (2020). Proinflammatory cytokines in Parkinson's disease and current treatment approaches. GSC Advanced Research and Reviews, 2(1), 21-25. 